\title{
INTEREST OF BEAM QUALITY IN MATERIALS TREATMENTS BY HIGH POWER LASER
}

\author{
D.F. GREVEY, K.F. BADAWI, J.P. BOQUILLON* and B. TAISNE \\ I.U.T. du Creusot, Laboratoire de Thermomécanique, 12 rue de la Fonderie, F-71200 Le Creusot, France \\ ${ }^{*}$ Université de Bourgogne, Laboratoire S.M.I.L., BP. 138, F-21004 Dijon cedex, France
}

\begin{abstract}
The aim of the study is the increasing of performances of the YAG Lasers. For this, we use the principle established by Boquillon and Taisne : an $X$ geometry of the resonator. In this case the number of emitted modes is lower than for the linear conception and the beam quality is greater. So, reached power density can be 7 times higher than for the classical cavity and consequently cutting speed can be about 2 times higher than for the linear one. Concerning welding, thick materials need a lot of energy and our system is not competitive. however it exists an interest for thin joining with an easely formed key hole and later a more important penetration.
\end{abstract}

\section{Introduction}

Numerous are the works which aim at increasing the obtained perfomances of materials treatments by high power laser. All parameters able to influence the efficiency of the laser matter efficiency are concerned.

With these parameters we can discriminate the power. Generally the aim of the studies that are made is to increase mean power in order to grows the depth of the treatment or the scaning speed. However for a given mean power, the only increase of power density allows an increase of performance. In this paper we propose results coming from this idea.. Power density is increased after the beam quality is ameliorate owing to the Boquillon and Taisne's principle [1].

\section{Experimental Device}

The basic installation is a type LAP 4000 YAG Laser from Laser Application. We use it according two configurations :

- with a classical linear resonator (figure 1). It allows to deliver a maximal mean power of $450 \mathrm{~W}$.

- with an "X resonator" (figure 2). It's a stable ring resonator, with 4 mirors, imagined in view to reinforced the oscillation in a privileged direction owing to a $5^{\text {th }}$ miror which reflects the wave on it's way. This resonator permits to increase the pulse energy with the same electrical power. We have also a greater reproductibility in the pulses because of the decrease of the hole burning phenomenon. The principle of this resonator is patented [1]. The maximal mean power reached with our installation (LAP 4000) is about 300W. 
Obtained characteristics with these 2 types of sources are shown on figure (3) and (4). The figure (3) is relative to the beam quality factor or Lagrange-Smith invariant [3] and the figure (4) illustrate maximal power density reached in the focal area after a plano convex optic (focal length $=80 \mathrm{~mm}$ ). We can see that the Lagrange- Smith invariant is lower in the case of the " $X$ resonator" than for the other one. This result is due to a lower divergence in the first configuration. (see table I)

\begin{tabular}{|c|c|c|}
\hline Power (W) & $\begin{array}{c}\text { Divergence } \\
\text { linear cavity (mrad) }\end{array}$ & $\begin{array}{c}\text { Divergence } \\
\text { X cavity (mrad) }\end{array}$ \\
\hline 100 & 10,37 & 4,99 \\
100 & 12,05 & 5,43 \\
\hline 200 & 13,30 & 7,36 \\
300 & 14,1 & 12,64 \\
\hline
\end{tabular}

Therefore power density are greater for the $X$ cavity than for the linear cavity because of a more little beam waist after focusing optics (if all other parameters are equal).

Effectively for a given mean power, the "X resonator" allows to obtain power densities 7 times higher than the linear resonator (in certain case 20 times higher [2]).

So we can think that this type of resonator is interesting for certains industrial applications like cutting or welding.

\section{Comparison of the two resonators for the cutting process}

In view to compare the two configurations of resonators we hold constant all the concerned parameters :

- the geometry of the cutting head, it's position in comparison with the surface of the sample, the nature (air) and flow of cutting gas.

- the operational parameters : energy, duration and frequency of pulses for a given mean power.

- the laser divergence after expander ( 2 to $4 \mathrm{mrad})$

- the spot diameter on focusing optics (about 15mm)

Obtained results for stainless steel cutting are presented on figure 5 and 6 . We can see that scanning speed are about 2 times higher with the $X$ resonator than with the other one.

For the lowest power (20W), the cutting speed is limited by the frequency of pulses with the $X$ cavity. In opposite, with the linear system it is not possible to cut the steel through lack of power density.

If we use the linear cavity in cutting configuration (the back miror is moving away from the rod) we obtain the same results in cutting speed than for the $X$ cavity. But maximal mean power is limited to $180 \mathrm{~W}$ with the linear cavity in cutting configuration and can reached $300 \mathrm{~W}$ for the $\mathrm{X}$ resonator. The advantage of the last one appears clearly. 


\section{Comparison of the two configurations for the welding process}

The welding needs energy for material melting and power density in view to the formation of the key hole. With our system we can't have more energy than with the linear configuration. So we will be limited in penetration but we can ameliorate the geometry of the melted zone owing to the high level of power density. Results are illustrated on figure 7 and 8 . On the 7 th figure we have a micrography of the melted zone obtained with the linear resonator. The geometry of the melted zone is governed by conduction laws, and the penetration is about $0,48 \mathrm{~mm}$ for $100 \mathrm{~W}$ of mean power. The micrography 8 shows that a key hole was formed and consequently the penetration is higher and reached $1,2 \mathrm{~mm}$.

\section{Conclusion}

We have seen that it is possible to increase performance of YAG laser owing to a special configuration of the resonator, named "X cavity". The better performances are due to a lower divergence of the beam and consequently allows to have higher power density than for the linear configuration.

The applications which are interested by this system are those who need high power density, like cutting, drilling... Concerning welding, if penetrations are limited because of the lack of energy, it's easier to form a key hole and to decrease the heat affected zone.

\section{BIBLIOGRAPHY}

[1] BOQUILLON [J.P.]; TAISNE [B.]

Demande de brevet $n^{\circ} 89.15627$ du 28/11/89, $n^{\circ}$ Publication 2.625.211 du 31/05/91

[2] GREVEY [D.F.] et al.

Etude des caractéristiques d'une cavité Laser en $\mathrm{X}$ de forte puissance

Soumis à "Journal de Physique III"

[3] BADAWI [K.F.] et al.

Revue de "Physique appliquée", n²5, Mars 1990, pp. 313-321 


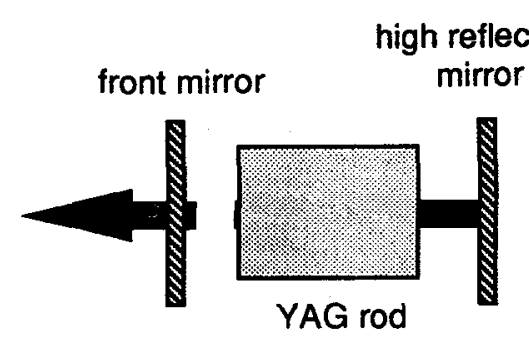

figure 1: linear configuration of the cavity

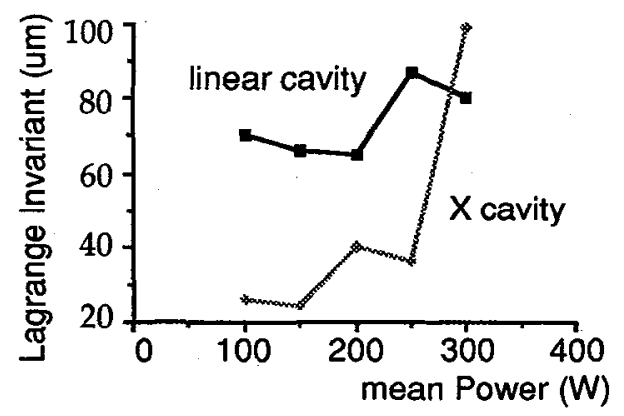

figure 3: variation of the beam quality factor (Lagrange Invariant) versus mean power.

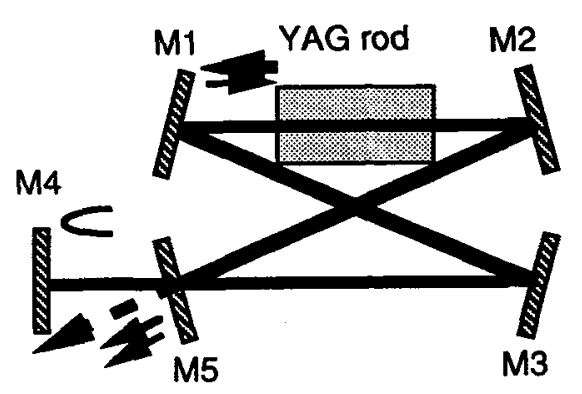

figure 2: geometry of the $X$ cavity

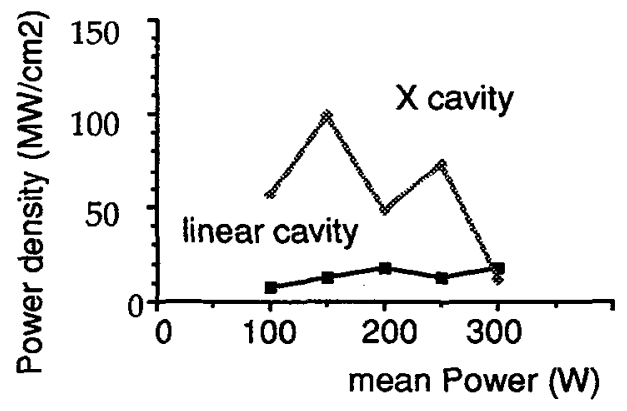

figure 4: power density versus mean power (in the focal zone of a $80 \mathrm{~mm}$ foc.)
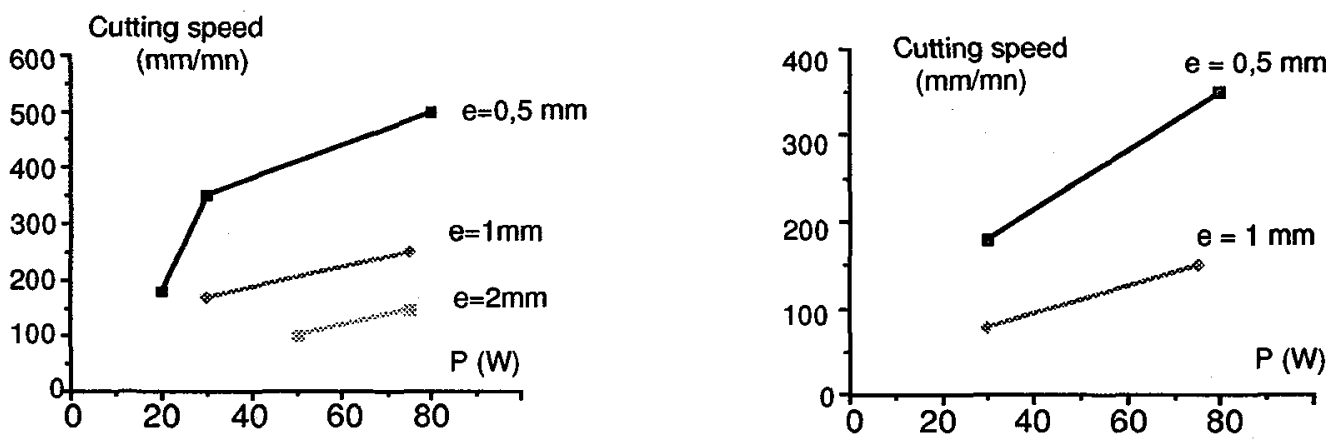

figure 5: cutting speed obtained for a stainless steel with the $X$ resonator

figure 6: obtained speed for stainless steel cutting with the linear cavity 